\title{
Proximal epithelioid sarcoma of the vulva: A case report and re- view of the literature
}

\author{
Mercedes Bravo-Taxa ${ }^{a,}{ }^{,}$, Luis Taxa-Rojas ${ }^{a}$, Aldo López-Blanco ${ }^{b}$ \\ a Pathology Department, Instituto Nacional de Enfermedades Neoplásicas (INEN), Lima, Peru. \\ ${ }^{b}$ Surgical Gynecology Department, Instituto Nacional de Enfermedades Neoplásicas (INEN), Lima, Peru.
}

\begin{abstract}
Primary sarcomas of the vulva are rare, with an incidence of $1.5 \%$ to $5 \%$ of all vulvar malignancies. There are only 41 cases described in the English literature of proximal epithelioid sarcoma of the vulva, which also constitutes a highly aggressive neoplasm with a high rate of recurrence and metastasis. We are reporting the first case of vulvar epithelioid sarcoma reported in our country, Peru, and Latin America. We present the case of a 53-year-old woman with no significant history who presented a large tumor mass of $25 \mathrm{~cm}$ in the left hemivulva of 2 years of evolution, associated with intense pain. She underwent radical surgical resection with left inguinopelvic lymphadenectomy and reconstruction of Rectus Abdominal Muscle Myocutaneous Flaps (TRAM).

Keywords: Epithelioid sarcoma; vulva; sarcoma
\end{abstract}

\section{INTRODUCTION}

Vulvar sarcoma constitutes $1.5-5 \%$ of all malignancies in this gynecologic region ${ }^{[1-5]}$. Types of sarcomas commonly present in the vulva include leiomyosarcomas, malignant fibrous histiocytoma, malignant rhabdoid tumor, angiosarcoma, rhabdomyosarcoma, and epithelioid sarcomas ${ }^{[1-3]}$. Epithelioid Sarcoma is subclassified into distal or proximal type about the involvement of upper and lower extremities or the pubic region and trunk, respectively. Proximal type epithelioid sarcoma of the vulva is an exceedingly rare soft tissue tumor, with only 41 cases described in the English literature, and extremely aggressive with a high rate of local recurrence and distant metastasis ${ }^{[2,4]}$. The following

* Corresponding author: Mercedes Bravo-Taxa

Mailing address: Calle Las Dalias 351, La Molina, Lima 12, Perú.

E-mail: mercedes.bravo.t@upch.pe

Received: 25 June 2021 / Accepted: 24 August 2021 publication provides the first case of vulvar epithelioid sarcoma reported in our country, Peru and Latin America; and reviews its clinical, histomorphological, treatment, and prognosis of this unusual pathology.

\section{CASE PRESENTATION}

A 53-year-old woman presented with a palpable progressive growth tumor in the left hemi-vulva for 2 years, which became more abrupt in the last 6 months and generated permanent intense pain. Upon examination, an $18 \mathrm{~cm}$-sized tumor was seen on the labia majora of the left hemi-vulva, without infiltration of the urethral meatus or vaginal introitus; however, it compromised the adjacent skin and presented a $2-3 \mathrm{~cm}$ sized left inguinal lymphadenopathy. The coronal and axial MRI images revealed the presence of a $17.5 \times 15 \times$ $15.3 \mathrm{~cm}$ extensive solid lesion with polylobulated margins and necrotic component located in the left vulvar region, infiltrating fatty planes, and conditioning effect of mass on the adductor muscles fascia of the left thigh. In addition, lymphadenopathy was identified in the left inguinal region, external iliac chain, and left obturator 
group (Figure $1 \mathrm{~A}, \mathrm{~B}$ ). Chest and brain CT were negative for metastases.

In November 2020, the multidisciplinary team decided to perform radical vulvar resection plus selective left inguinal and pelvic lymphadenectomy, as well as the reconstruction with a Rectus Abdominal Muscle Myocutaneous Flaps (TRAMP).

Gross examination showed a 33 × $30 \times 14 \mathrm{~cm}$-sized multilobed indurated tumor in the left hemi-vulva , extending to the vaginal introitus, suprapubic region, and bilateral inguinal region, predominantly on the left side (Figure $2 \mathrm{~A}, \mathrm{~B}, \mathrm{C}, \mathrm{D})$ The sectional surface was solid, heterogeneous with intermingled tan- whitish, myxoid, necrotic, hemorrhagic, and cystic degeneration areas.

The microscopic appearance of the tumor was composed of long polygonal spindle cells with abundant eosinophilic cytoplasm, pleomorphic vesicular nucleus, prominent nucleolus, and high mitotic activity, $22 \mathrm{mi}-$ toses per 10 high-power fields. Likewise, lymphovascular and lymph node invasion was identified. 14 of 19 inguinocrural lymph nodes and 5 of 7 iliac lymph nodes were compromised with the epithelioid sarcoma. The surgical margins were free of injury (Figure 3). The immunophenotype of the neoplastic cells showed immunoexpression for Pankeratin, EMA, Vimentin, Actin; while they were negative for Desmin, CD10, CD34, CD138, Melan A, HMB45, SOX-10, S100, Estrogen receptor, and CDX2. And the most relevant immunohistochemical finding, that determined the diagnosis, was the loss of INI-1 expression, concluding that the tumor corresponded to a Proximal Epithelioid Sarcoma of the vulva (Figure 4).

With this result, the patient was tributary to adjuvant treatment with radiotherapy and chemotherapy. She received 3 cycles of chemotherapy with Isofosfamide / Adriamycin. Subsequently, by July 2021, she completed 35 treatment cycles of external radiotherapy at a dose of $45000 \mathrm{~Gy}$ to the pelvic and inguinal region using the Volumetric modulated arc therapy (WMAT) from $6 / 23 / 21$ to $7 / 25 / 21$ with excellent tolerance. Currently, she remains without evidence of recurrence after 9 months of follow-up, with the possibility of receiving more cycles of chemotherapy.

\section{DISCUSSION}

Vulvar cancer is the fourth most common cause of gynecological malignancy, constituting $5 \%$ of all cancers of the female genital tract ${ }^{[1,2]}$, Among the various histological types of vulvar cancer, squamous cell carcinoma is the most common type and represents $95 \%$ of all malignant neoplasms in this gynecological area, followed by melanoma, sarcoma, and basal cell carcinoma ${ }^{[1]}$. Primary vulvar sarcomas are rare, reaching an incidence of 1.5 to $5 \%$ of cancers in this topographic region ${ }^{[2,3]}$.

Epithelioid sarcoma (ES) is a malignant soft tissue neoplasm of uncertain histogenesis, which was established as a distinctive disease by Ezinger in $1970{ }^{[3]}$. Epithelioid sarcomas (ES) are classified into distal, conventional, or classic type and proximal type based on its occurrence in the upper and lower or in the trunk and pubic region, respectively ${ }^{[2-4]}$. The proximal epithelioid sarcoma of the vulva are rare entities, with only 41 cases published in the English literature, and the first of them described by Piver et al. in $1972^{[2,4]}$ (Table 1).

It occurs more frequently in women of reproductive age with a mean age of 37.8 years and mainly affects the labia majora, followed by the Bartholin gland, clitoris, and
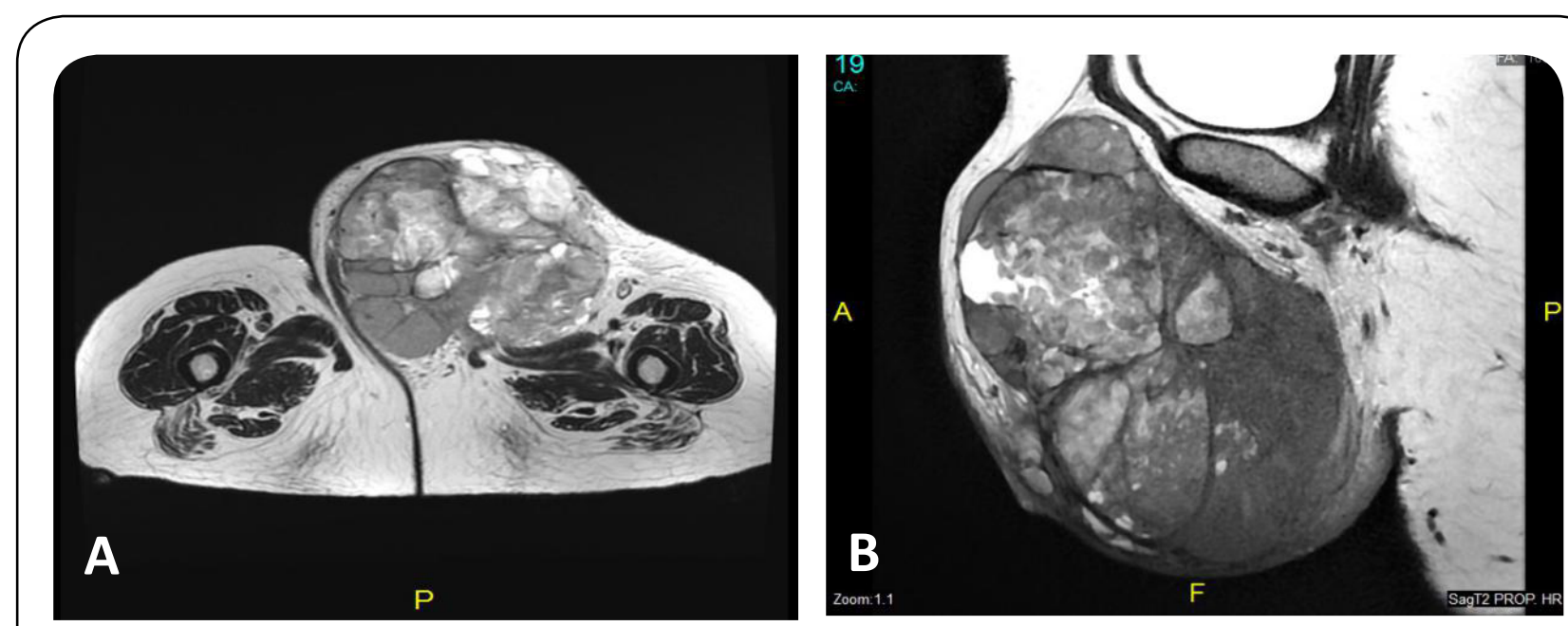

Figure 1. (A) Axial and (B) Coronal MRI images with an extensive solid lesion with polyobulated margins and necrotic component located in left vulvar region and infiltrating fatty planes. 

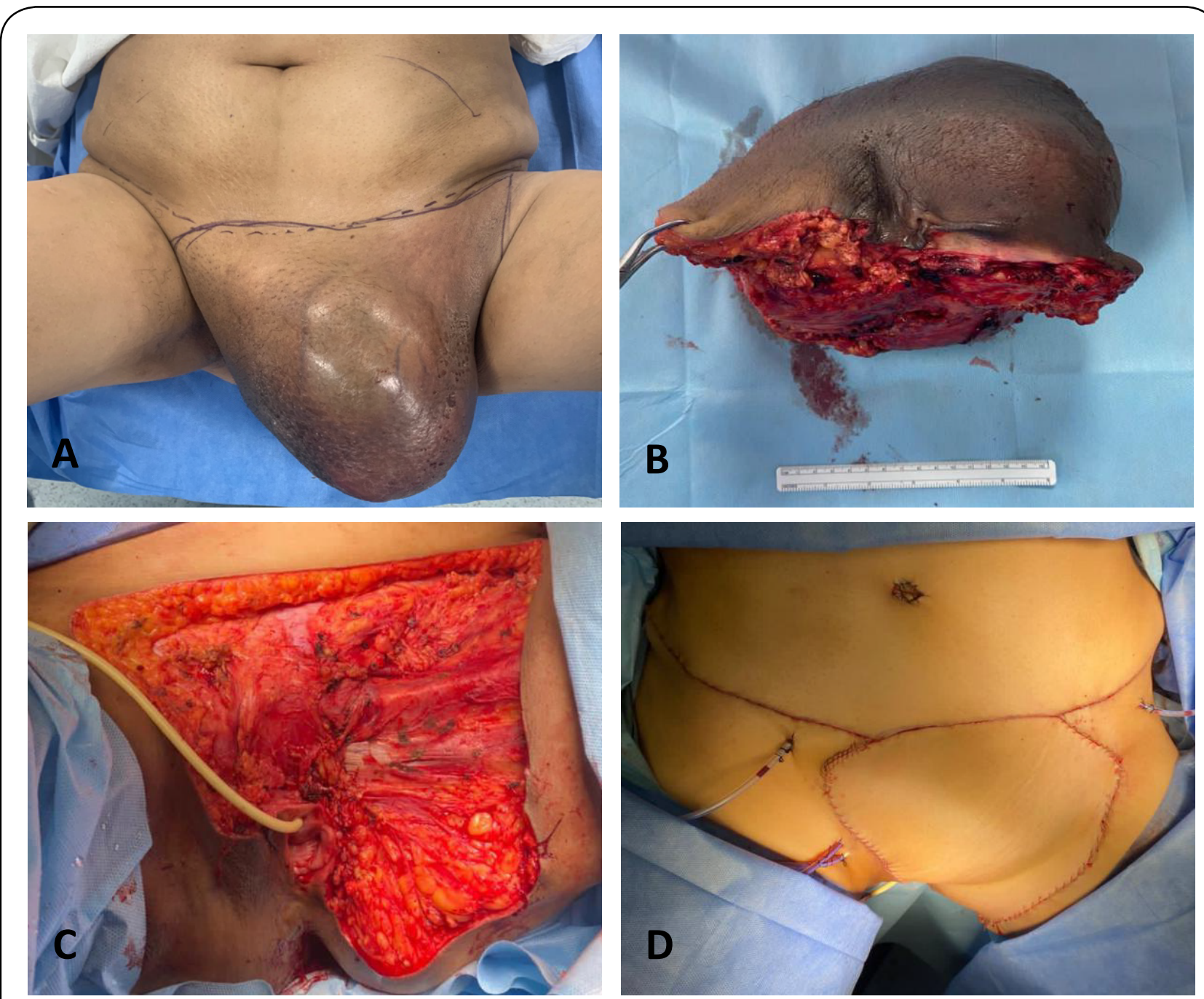

Figure 2. A-B: Extensive solid lesion in left hemi-vulva, which extends to the vaginal introitus, suprapubic region and bilateral inguinal region. C: Radical resection plus left inguino-pelvic lymphadenectomy. D: Simultaneously, reconstruction was performed with an abdominal myocutaneous flap.

labia minora. The most common initial clinical symptom is the presence of a slow-growing painless mass on the labia majora, which makes the diagnosis more difficult and delayed since they are often confused with Bartholin cysts, lipomas, or genital warts ${ }^{[2-4]}$. In our case, the lesion affected the labia major and presented as a palpable progressive mass regarded as a benign condition for the patient, so adequate treatment was delayed for 2 years without any treatment.

The microscopic appearance of Epithelioid sarcoma (ES) differs based on the type of distal o proximal tumor is the one affecting.

Classic or conventional, the distal type usually shows as a dermal or subcutaneous nodule. It also can simulate granulomatous process, with or without necrosis (pseudogranulomatous morphology). Tumor cells are fairly uniform plump small to medium-sized cells with eosinophilic cytoplasm, spindle cells can also be identi- fied and often appear more conspicuous at the periphery of a nodule. Nuclei show a mildly atypical appearance with vesicular chromatin and small nucleoli. The mitotic activity is variable, mixed chronic inflammatory infiltrates common to identified, dystrophic calcification and metaplastic bone formation can also be seen ${ }^{[6]}$. On the other hand, the proximal type shows an infiltrative growth pattern with less defined peripheral borders and multiple large nodules and/or in sheets pattern. The tumor cells are large polygonal cells with abundant eosinophilic or amphophilic cytoplasm, rhabdoid morphology is common. Nuclei show vesicular chromatin with prominent macronuclei. High mitotic activity, necrosis, and hemorrhage are common ${ }^{[2-6]}$. Also, an unusual pattern has been recognized in proximal ES of the vulva, which may present myxoid changes ${ }^{[3]}$. Our case report showed a similar microscopic appearance as the literature report, the tumor was composed of 


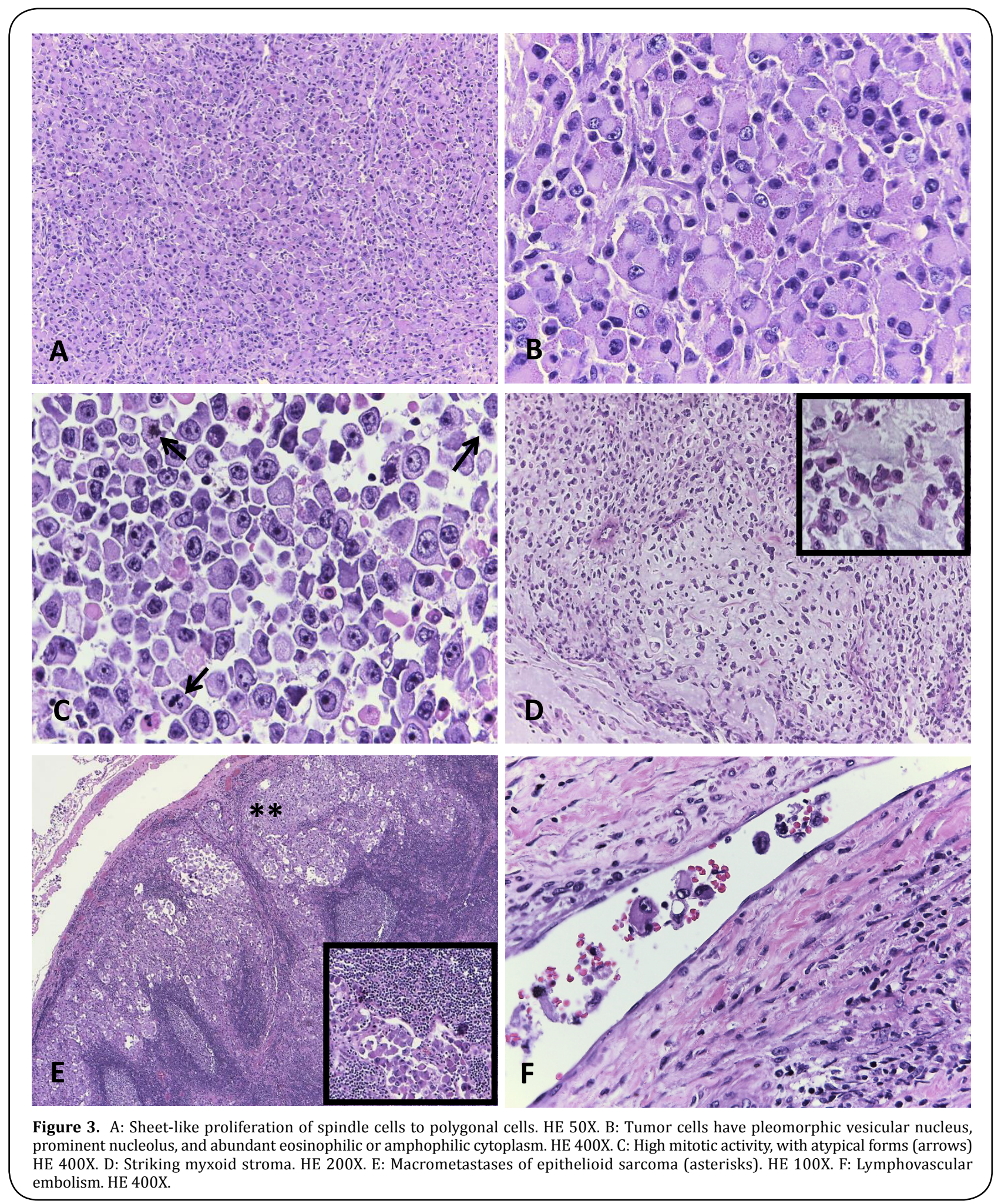

long polygonal spindle cells with abundant eosinophilic cytoplasm, pleomorphic vesicular nucleus, prominent nucleolus, and high mitotic activity, 22 mitoses per 10 high-power fields Also we recognized areas of myxoid changes, something not usual, in this type of tumor, but there is a report of this rare pattern. The diagnosis requires the support of the immunohis- tochemical profile. Epithelioid sarcoma (ES) reveals cytoplasmic immunoreactivity for keratin, vimentin, and EMA, while neoplastic cells are negative for S-100 and CD31 ${ }^{[5,7]}$. On the other hand, an essential marker to confirm the diagnosis of Epithelioid sarcoma (ES) is the absence of INI1 expression ${ }^{[8]}$. The neoplastic cells, of our case report, showed immunoexpression for Pan- 
keratin, EMA, Vimentin, Actin; while they were negative for Desmin, CD10, CD34, CD138, Melan A, HMB45, SOX-10, S100, Estrogen receptor, and CDX2 and lack of staining for INI-1 protein.

This immunophenotypic expression pattern allows us to differentiate Epithelioid sarcoma (ES) from other soft tissue malignancies with epithelioid appearances such as synovial sarcoma, extrarenal malignant rhabdoid tumor, malignant epithelioid peripheral nerve sheath tumor, melanoma, rhabdomyosarcoma, and undifferentiated carcinoma ${ }^{[3-8]}$.

The SMARCB1 gene located on chromosome 22q11 acts as a tumor suppressor gene. However, recent studies have shown the loss of expression of this protein, INI1/SMARCB1, in more than $90 \%$ of cases of Epithelioid sarcoma (ES), which plays a crucial role in its tumorigenesis ${ }^{[3,4,9]}$. The complete absence of INI1/ SMARCB1, which is a tumor suppressor belonging to

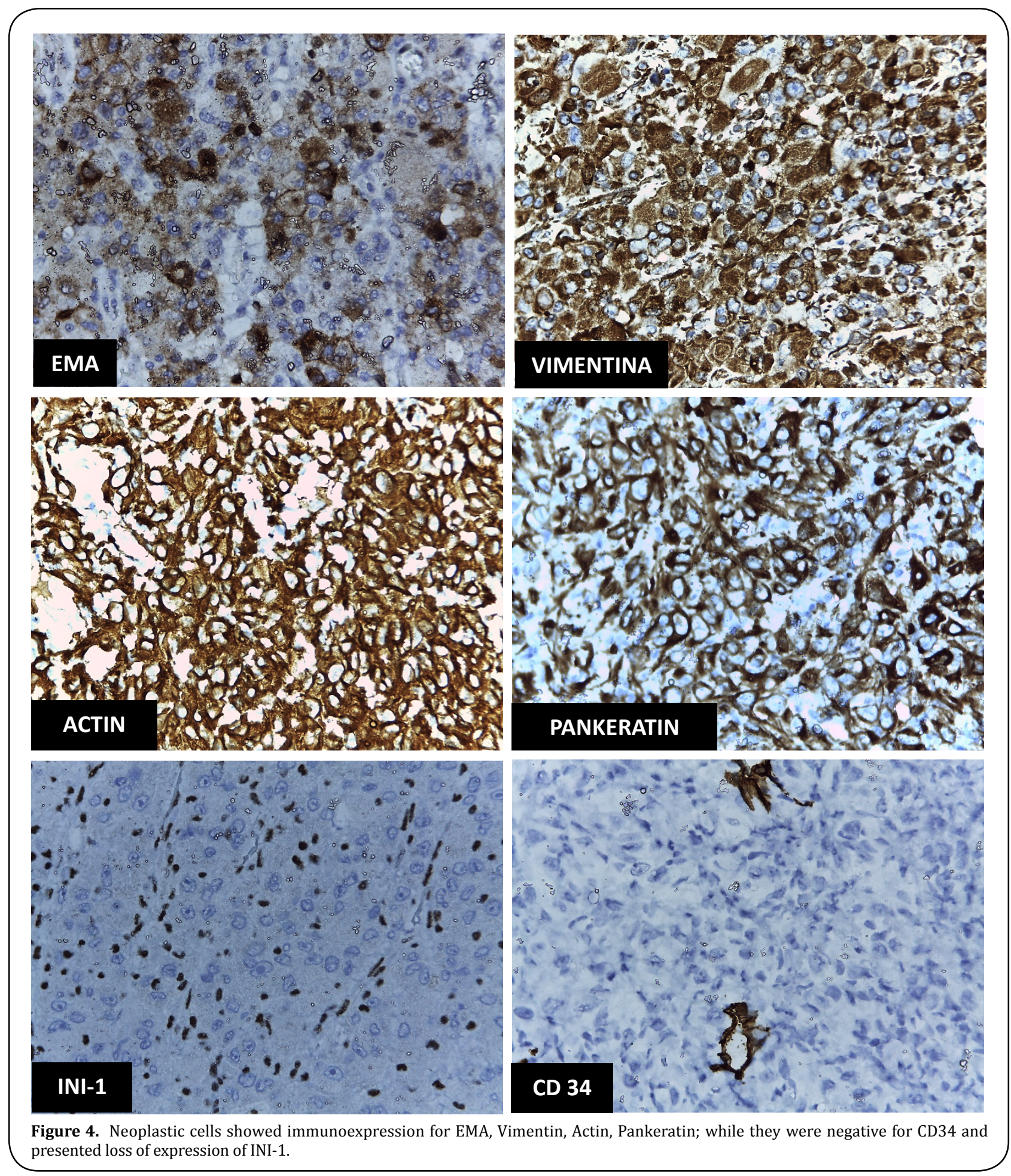


the SWI/SNF complexes, leads to elevated expression of EZH2, a component of polycomb repressive complex 2, which mediates gene silencing by catalyzing H3K27me ${ }^{[3,10,11]}$. Tazemetostat is a new oral compound able to inhibit EZH2, whose blockade prevents the methylation of histone H3K27, thus decreasing the growth of EZH2 mutated or over-expressing cancer cells, therefore neutralizing this effect. Tazemetostat has been investigated in a Phase II study and has shown clinical activity in INI1-negative Epithelioid sarcoma (ES) in approximately $15 \%$ of the patients, with durable responses and an overall tolerable safety profile. Based on these results, the U.S. Food and Drug Administration (FDA) has approved tazemetostat for adults and pediatric patients aged 16 years and older with metastatic or locally advanced Epithelioid sarcoma (ES) not eligible for complete resection ${ }^{[12]}$. Tazemetostat represents a new treatment option for Epithelioid sarcoma (ES) patients, although clinical/ molecular predictors of response are still to be identified. The combination of tazemetostat with other drugs like doxorubicin and immunotherapeutic agents is currently under investigation in Epithelioid sarcoma (ES) patients ${ }^{[13]}$.

The ideal treatment remains uncertain; however, the mainstay of initial management is surgical resection with wide negative margins of more than $2 \mathrm{~cm}^{[2-4]}$.

Loco-regional lymph node dissection is controversial, Kim et al. propose the dissection of enlarged nodes, even though, there is no evidence that it has a significant impact on local or distant relapse ${ }^{[5]}$. In contrast, Kasumatsu et al. suggest that radical resection and inguinal dissection is the best choice since it offers better control of disease and could postpone adjuvant treatment ${ }^{[7]}$. The treatment decided to perform in our case report radical vulvar resection plus selective left inguinal and pelvic lymphadenectomy, as well as the reconstruction with a Rectus Abdominal Muscle Myocutaneous Flaps (TRAM).

The role of adjuvant radiotherapy and/or chemotherapy is not clear. Some reports advocate the use of radiotherapy in high-grade tumors or those with inadequate surgical margins, and there have even been cases of a decrease in the rate of recurrence in patients who received this type of adjuvant ${ }^{[14,15]}$. Meanwhile, chemotherapy effectiveness is not clear, there is literature with very varied results and rates of recurrence, with minimal benefits from its use; however, due to the low number of cases available, chemotherapy remains an alternative treatment ${ }^{[3,4,14]}$. In our case, the patient received adjuvant radio-chemotherapy after surgical resection. She received 3 cycles of chemotherapy with Isofosfamide / Adriamycin. Subsequently, she completed 35 treatment cycles of external radiotherapy at a dose of 45000 Gy to the pelvic and inguinal region using the Volumetric modulated arc therapy (WMAT) from $6 / 23 / 21$ to $7 / 25 / 21$ with excellent tolerance. Currently, she remains without evidence of recurrence after 9 months of follow-up, with the possibility of receiving more cycles of chemotherapy depending on the CT scan controls.

The prognosis of Epithelioid sarcoma (ES) is not favorable, the lack of symptoms added to its aggressive nature with a rapid tendency to metastasis and high recurrence rate, despite the presence of wide negative margins, cause a not very long survival in these patients. The overall survival rate is 21 months for the initial stages and 6 months for the advanced stages of the disease ${ }^{[2-4]}$. Locoregional lymph node involvement, vascular invasion, tumor size greater than $2 \mathrm{~cm}$, depth of involvement, presence of necrosis, and high mitotic index, exceeding 2 mitoses per 10 high-power fields, are considered as poor prognostic factors ${ }^{[3,5]}$.

According to reports in the literature, 38 to $69 \%$ of patients develop recurrence, which usually occurs within the first 6 months of the initial treatment, the most common sites of involvement being the lungs and lymph nodes. Furthermore, $62.5 \%$ of patients who develop a recurrence will die from the disease ${ }^{[2-4]}$. In our case, after 9 months of the surgical resection and follow-up, she remains without evidence of recurrence or adverse effects for the adjuvant therapy.

\section{CONCLUSION}

Proximal epithelioid sarcomas of the vulva are an extremely aggressive condition with a high tendency to recurrence and metastasis, so a timely diagnosis with surgical resection and wide negative margins is necessary.

Data related to the tumor is limited; therefore, it is particularly important to encourage prospective and randomized clinical trials that are focused on the treatment of the tumor and to determine the efficacy of possible adjuvant therapies as Tazemetostat, which seems to be the promising cure for advanced cases.

\section{DECLARATIONS}

\section{Authors' contributions}

Dr. Bravo analyzed the clinical data and drafted the 
Table 1. Published cases with vulval epithelioid sarcoma.

\begin{tabular}{|c|c|c|c|c|c|c|c|c|}
\hline First autor, country & Year & Age & Surgery & LND & ADJ & $\begin{array}{l}\text { DFS } \\
\text { (months) }\end{array}$ & $\begin{array}{l}\text { OS } \\
\text { (months) }\end{array}$ & Outcome \\
\hline Bravo, Perú & 2021 & 53 & Wide local excision & B & RT & 2 & 2 & NED \\
\hline Jeon, Korea & 2020 & 77 & Wide local excision & B & RT & - & 36 & DOD \\
\hline Yue, China & 2018 & 41 & Wide local excision & - & - & 9 & 9 & NED \\
\hline Chan Hee Han, USA & 2016 & 32 & Wide local excision & - & - & 9 & 9 & NED \\
\hline Rego, Brazil & 2015 & 34 & Wide local excision & - & - & - & 18 & NED \\
\hline Patrizi, Italy & 2013 & 63 & Radical vulvectomy & B & RT & - & 14 & NED \\
\hline Kim, Korea & 2012 & 41 & Local excision & - & RT & - & 10 & NED \\
\hline Ong, Sigapore & 2012 & 51 & Hemivulvectomy & Y & - & - & 8 & NED \\
\hline Andrisani, Italy & 2011 & 46 & Radical vulvectomy & B & RT, CT & 1 & 12 & DOD \\
\hline Chiyoda, Japan & 2011 & 33 & Radical vulvectomy & B & - & - & 36 & NED \\
\hline Manzanares-Campillo, Spain & 2011 & 57 & Local excision & - & - & 48 & 96 & NED \\
\hline Tholpady, USA & 2010 & 17 & Local excision & - & - & - & 12 & NED \\
\hline Rai, USA & 2009 & 17 & Local excision & B & RT & 2 & 2 & AWD \\
\hline Mas-Morey, Spain & 2008 & 30 & Wide Local excision & B & RT & - & 12 & NED \\
\hline Eroglu, Turkey & 2008 & 25 & Wide local excision & Y & RT & - & 16 & NED \\
\hline Kim, Korea & 2008 & 24 & Wide local excision & - & - & - & 8 & NED \\
\hline Argenta, USA & 2007 & 35 & Radical excision & Y & RT & - & 40 & NED \\
\hline Dainese & 2005 & 34 & Radical hemivulvectomy & Y & - & - & 12 & NED \\
\hline Altundag, Turkey & 2004 & 51 & Hemivulvectomy & B & RT, CT & 4 & 6 & DOD \\
\hline Ulutin, USA & 2003 & 42 & Radical vulvectomy & - & - & - & 146 & NED \\
\hline Moore, USA & 2002 & 29 & Radical vulvectomy & - & $\mathrm{CT}$ & 6 & 6.5 & DOD \\
\hline Gonzalez-Peramato, Spain & 2001 & 84 & Local excision & - & $\mathrm{CT}$ & 1 & 1 & AWD \\
\hline Kasamatsu, Japan & 2001 & 23 & Local excision & - & - & 30 & 96 & NED \\
\hline Hasegawa & 2001 & $\begin{array}{l}37 \\
30 \\
80\end{array}$ & $\begin{array}{l}\text { Local excision } \\
\text { Local excision } \\
\text { Wide local excision }\end{array}$ & $\begin{array}{l}- \\
- \\
-\end{array}$ & $\begin{array}{l}\text { CT } \\
- \\
-\end{array}$ & $\begin{array}{l}- \\
96 \\
10\end{array}$ & $\begin{array}{l}11 \\
104 \\
23\end{array}$ & $\begin{array}{l}\text { NED } \\
\text { NED } \\
\text { DOD }\end{array}$ \\
\hline Tjalma, Belgium & 1999 & 23 & Hemivulvectomy & Y & - & - & 48 & NED \\
\hline & & 57 & Local excision & - & RT & - & 52 & DOID \\
\hline Guillou, Switzerland & 1997 & $\begin{array}{l}49 \\
45\end{array}$ & $\begin{array}{l}\text { Radical vulvectomy } \\
\text { Wide local excision }\end{array}$ & - & $\begin{array}{l}\text { RT } \\
\text { RT, CT }\end{array}$ & - & $\begin{array}{l}22 \\
21\end{array}$ & $\begin{array}{l}\text { NED } \\
\text { NED }\end{array}$ \\
\hline Ross, USA & 1997 & 20.5 & Local excision & - & - & - & 105 & NED \\
\hline Hernandez-Ortiz, Spain & 1995 & 51 & Radical vulvectomy & - & - & 11 & 12 & DOD \\
\hline Konefka, Poland & 1994 & 49 & Radical vulvectomy & B & $\mathrm{CT}$ & 5 & 8 & DOD \\
\hline Weissmann, USA & 1990 & 26 & Local excision & - & - & 14 & 78 & AWD \\
\hline Perrone, USA & 1989 & 21 & Local excision & Y & - & - & 56 & NED \\
\hline Tan, Singapore & 1989 & 21 & Local excision & - & - & - & 36 & NED \\
\hline Wevers, Netherland & 1989 & 37 & Local excision & $\mathrm{Y}$ & - & 2 & 5 & DOD \\
\hline \multirow{2}{*}{ Ulbright, USA } & \multirow{2}{*}{1983} & 55 & Local excision & - & - & 8 & 15 & DOD \\
\hline & & 30 & Wide local excision & - & - & 1.5 & 8 & DOD \\
\hline Hall, USA & 1980 & 31 & Wide local excision & $\mathrm{Y}$ & - & 35 & 70 & DOD \\
\hline Gallup, USA & 1976 & 31 & Radical vulvectomy & - & - & - & 16 & NED \\
\hline Piver, USA & 1972 & 27 & Local excision & - & - & - & 108 & NED \\
\hline
\end{tabular}

LND: lymph node dissection, Y: yes, B: bilateral, RT: radiation therapy, CT: chemotherapy, NED: no evidence of disease, DOD: die of disease, AWD: alive with disease, DOID: die of interurrent disease, DFS: disease free survival, OS: overall survival. 
manuscript. Dr. Taxa provided the pathological results and provided professional guidance on this. Dr. López provided imaging studies and conducted follow up on the patient in the outpatient clinic. Dr. Taxa and Dr. López contributed to critical review and supervised the entire study. All the authors have read and approved the final version of this manuscript.

\section{Conflicts of interest}

All authors declared that there are no conflicts of interest.

\section{REFERENCES}

1. Siegel, R., Naishadham, D., \& Jemal, A. (2013). Cancer statistics, 2013. CA: A Cancer Journal for Clinicians, 63(1), 11-30.

2. Jeon, S. E., Lee, J., Jung, H. J., Kim, B. J., Ryu, S. Y., \& Park, S. I. (2020). An extremely rare elderly case of proximal epithelioid sarcoma of the vulva: case report with a review of literatures. Obstetrics \& Gynecology Science, 63(5), 670-674.

3. Rego, J. L., Cintra, G. F., Netto, A. K., Abrahão-Machado, L. F., \& Tsunoda A. (2015). Extremely rare case of vulvar myxoid epithelioid sarcoma. Case Rep Obstet Gynecol, 2015, 971217.

4. Han, C. H., Li, X., \& Khanna, N. (2016). Epithelioid sarcoma of the vulva and its clinical implication: A case report and review of the literature. Gynecologic Oncology Reports, 15, 31-33.

5. Kim, J. H., Choi, Y. S., \& Lee, T. S. (2008). A case of epithelioid sarcoma arising in the vulva. Journal of Gynecologic Oncology, 19(3), 202-204.

6. Fisher, C. (2006). Epithelioid sarcoma of Enzinger. Advances in Anatomic Pathology, 13(3), 114-121.

7. Kasamatsu, T., Hasegawa, T., Tsuda, H., Okada, S., Sawada, M., \& Yamada, T. (2001). Primary epithelioid sarcoma of the vulva. International Journal of Gynecological Cancer, 11(4), 316-320.
8. Kim, H. J., Kim, M. H., Kwon, J., Kim, J. Y., Park, K., \& Ro, J. Y. (2012). Proximal-type epithelioid sarcoma of the vulva with INI1 diagnostic utility. Annals of Diagnostic Pathology , 16(5), 411-415.

9. Noujaim, J., Thway, K., Bajwa, Z., Bajwa, A., Maki, R. G., \& Jones, R. L. (2015). Epithelioid Sarcoma: Opportunities for Biology-Driven Targeted Therapy. Frontiers in Oncology, 5, 186.

10. Modena, P., Lualdi, E., Facchinetti, F., Gall, L., Teixeira, M. R., \& Pilotti, S. (2005). SMARCB1/INI1 tumor suppressor gene is frequently inactivated in epithelioid sarcomas. Cancer Research, 65(10), 40124019.

11. Daigle, S., Stacchiotti, S., Schöffski, P., Villalobos, V., Cote, G., \& Chugh, R. (2018). Molecular characterization of epithelioid sarcoma (ES) tumors derived from patients enrolled in a phase II study of tazemetostat (NCT02601950). Annals of Oncology, 29, viii670.

12. Gounder, M., Schöffski, P., Jones, R. L., Agulnik, M., Cote, G. M., \& Villalobos, V. M. (2020). Tazemetostat in advanced epithelioid sarcoma with loss of INI1/ SMARCB1: an international, open-label, phase 2 basket study. Lancet Oncology, 21(11), 1423-1432.

13. Simeone, N., Frezza, A. M., Zaffaroni, N., \& Stacchiotti, S. (2021). Tazemetostat for advanced epithelioid sarcoma: current status and future perspectives. Future Oncology, 17(10), 1253-1263.

14. Argenta, P. A., Thomas, S., \& Chura, J. C. (2007) . Proximal-type epithelioid sarcoma vs. malignant rhabdoid tumor of the vulva: a case report, review of the literature, and an argument for consolidation. Gynecol Oncology, 107(1), 130-135.

15. Hasegawa, T., Matsuno, Y., Shimoda, T., Umeda, T., Yokoyama, R., \& Hirohashi, S. (2001). Proximaltype epithelioid sarcoma: a clinicopathologic study of 20 cases. Modern Pathology, 14(7), 655-663. 